PROFESIONALES Y HERRAMIENTAS PARA EL DESARROLLO LOCAL Y SUS SINERGIAS TERRITORIALES. EVALUACIÓN Y PROPUESTAS DE FUTURO IX Coloquio Nacional de Desarrollo Local del GTDL-AGE 

ANTONIO MARTÍNEZ PUCHE, XAVIER AMAT MONTESINOS, ISABEL SANCHO CARBONELL y DANIEL SANCHIZ CASTAÑO (EDS.)

\section{PROFESIONALES Y HERRAMIENTAS PARA EL DESARROLLO LOCAL Y SUS SINERGIAS TERRITORIALES. EVALUACIÓN Y PROPUESTAS DE FUTURO}

IX Coloquio Nacional de Desarrollo Local del GTDL-AGE

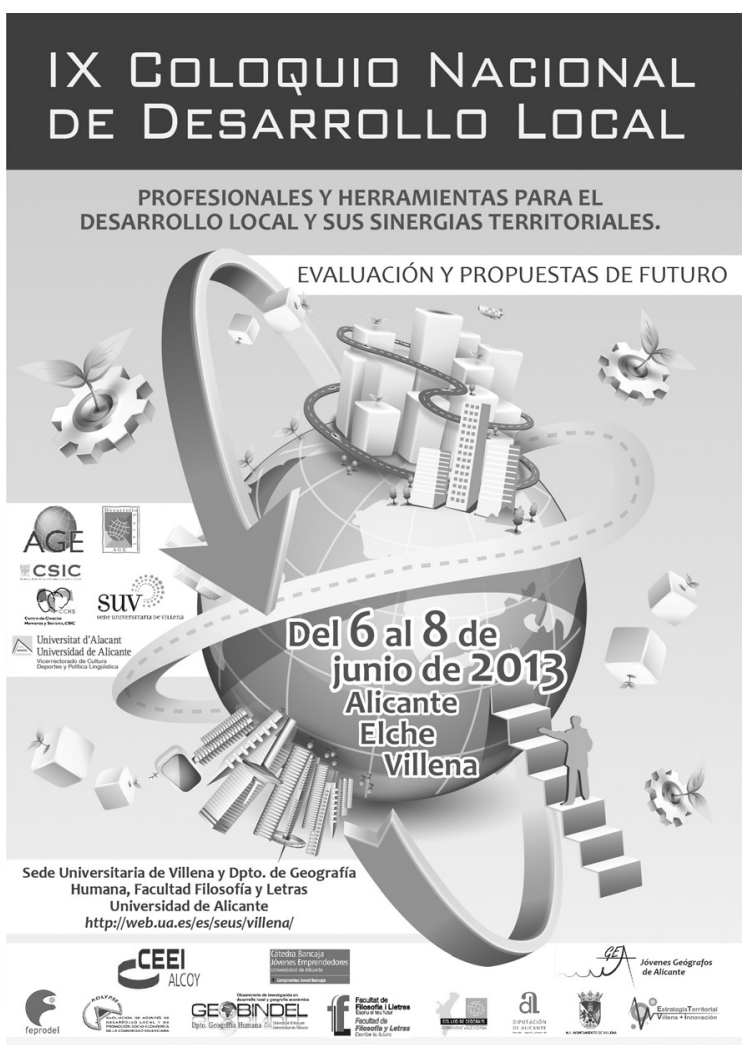


Este libro ha sido examinado y valorado por evaluadores ajenos a la Universidad de Alicante, con el fin de garantizar la calidad científica del mismo.

Publicacions de la Universitat d'Alacant

03690 Sant Vicent del Raspeig

Publicaciones@ua.es

http://publicaciones.ua.es

Telèfon: 965903480

(C) Antonio Martínez Puche, Xavier Amat Montesinos,

Isabel Sancho Carbonell y Daniel Sanchiz Castaño (eds.), 2016

(C) d'aquesta edició: Universitat d'Alacant

ISBN: 978-84-16724-00-0

Dipòsit legal: A 92-2016

Disseny de coberta: candela ink

Composició: Página Maestra (Miguel Ángel Sánchez Hernández)

Impressió i enquadernació: Guada Impresores

\section{unte \\ Unión de Editoriales
Universitarias Españolas \\ WWW.une.es
WWA}

Esta editorial es miembro de la UNE, cosa que garantiza la difusión y comercialización nacional y internacional de sus publicaciones.

Reservados todos los derechos. Cualquier forma de reproducción, distribución, comunicación pública o transformación de esta obra sólo puede ser realizada con la autorización de sus titulares, salvo excepción prevista por la ley. Diríjase a CEDRO (Centro Español de Derechos Repográficos, www.cedro.org) si necesita fotocopias o escanear algún fragmento de esta obra. 


\title{
EGRUYERE, OTRA FORMA DE GENERAR DESARROLLO LOCAL
}

\author{
María Gómez Javaloyes \\ Proyecto áSILO \\ maria.javaloyes@gmail.com \\ Ester Gisbert Alemany, \\ aRRsa! Plataforma Creativa \\ ester@arrsa.org \\ Sonia Beltrán Pastor \\ Reding, Estudio Creativo \\ beltran.pastor@gmail.com \\ Laura Yago Martínez \\ Reding, Estudio Creativo \\ laura@somosreding.com
}

RESUMEN

Un modelo de desarrollo local basado en el concepto de red distribuida, en la que todos los agentes implicados en el desarrollo económico y social de un contexto son considerados nodos igualmente generadores de desarrollo. $\mathrm{Y}$ esto es así porque se trabaja entre personas vinculadas a un hipercontexto, real y virtual que las conecta y que ellas pueden activar. Hablamos pues de lo concreto como un entorno real en el que trabajar y no de un espacio universal abstracto y que por tanto es inalcanzable.

Palabras clave: desarrollo local distribuido, redes distribuidas, hipercontexto, capital social, entorno real, nodos. 


\section{Abstract}

A local development model based on the concept of distributed network, in which all those involved in economic and social development of a context are also considered nodes generating development. And this is because it works between people linked to a hipercontexto real and virtual that they can connect and activate. We speak because of the concrete as a real environment in which to work and not an abstract universal space and therefore unattainable.

Key words: distributed local development, distributed networks, hipercontext, social capital, real environment, nodes.

\section{INTRODUCCIÓN}

Este coloquio tiene el objetivo de abrir la reflexión en torno al Desarrollo Local: el concepto, sus profesionales y sus herramientas. Algunos profesionales de la red eGruyere queremos aportar los conceptos que aplicamos en los proyectos de desarrollo local en que trabajamos. Aportación que hacemos desde nuestro perfil como emprendedores de micropymes y, por tanto, portadores y generadores de Desarrollo.

eGruyere es una red híbrida de profesionales que, desde 2010, comparten una visión renovada sobre lo laboral e intentan llevarla a la realidad de su trabajo, construyendo un espacio común -o una red de espacios- dónde poder hacerlo. Profesionales de diferentes disciplinas que, al estar conectadas y compartir filosofías de trabajo, construyen entre todas un valor de servicio colectivo (ayuda mutua, confianza, conocimiento, etc.) minimizando las necesidades organizativas y de medios de las estructuras clásicas de trabajo. Pero, sobretodo, eGruyère es algo vivo, que va evolucionando en el tiempo, poniendo ideas a prueba y aprendiendo de la experiencia.

Las autoras de esta comunicación son parte de esa red pero no hablan en nombre de ella como representantes de una institución que tenga un único discurso. En dicha comunicación, abordaremos diferentes conceptos. En primer lugar, hablaremos de las redes distribuidas, cuyos nodos conversan entre ellos como pares y ejercen diferentes roles al mismo tiempo y generan y atraen capital social. En según lugar, hablaremos de la Administración Pública como un nodo más que debe interactuar con más agentes. En tercer lugar, hablaremos del hipercontexto, una concepción del entorno que nos permite trabajar, no desde las fronteras geopolíticas, sino desde los vínculos reales entre las personas y sus conexiones translocales. Y en cuarto lugar, hablaremos de lo concreto frente a lo universal como planteamiento final y resultado de las reflexiones entorno a los conceptos anteriores. Expondremos también cuatro casos de estudio impulsados por nosotros y que serán analizados desde los cuatro enfoques anteriores. 
Nos gustaría remarcar que esta comunicación no pretende ser un modelo académico que pueda ser replicable en cualquier momento y lugar. Así mismo, tampoco pretende sustituir a los modelos establecidos por el nuestro, ya que, tampoco es nuestra finalidad aspirar a ser el modelo dominante. Esta comunicación pone de manifiesto nuestra nuestra visión y experiencia sobre Desarrollo Local, la que estamos probando y nos funciona a nosotros.

Una idea fundamental, si no la clave de todo, es que nuestra propuesta se basa en nuestra experiencia y en la conversación abierta que desarrollamos a través de herramientas conversacionales, fundamentalmente desde nuestros blogs personales.

\section{Un DESARROLLO LOCAL MÁS DistRIBUido}

Para defender la idea de un Desarrollo Local más distribuido, primero definiremos qué entendemos por Desarrollo Local distribuido, y luego justificaremos por qué nos parece una muy buena opción para todos aquellos interesados en este ámbito de trabajo, y justamente ahora.

Como Desarrollo Local distribuido entendemos aquel que se genera a través de unos nodos (profesionales u organizaciones) que no están necesariamente dentro de la Administración Pública ni forman parte de un sistema jerárquico donde los roles y responsabilidades están mediados por nadie. Como comentaremos más adelante, Desarrollo Local puede ser generado por aquellas personas con una motivación a hacerlo, con un conocimientos y unas afinidades relativas a un entorno real y concreto. El Desarrollo Local lo hacen las personas, que son las que poseen el conocimiento, la herramienta base para generar Desarrollo Local.

Las condiciones para afrontar el Desarrollo Local de esta manera son mejores que nunca, debido a los los recortes presupuestarios que se están experimentando y los que están por venir. Este contexto de crisis sólo hace que poner en jaque esta forma centralizada e incluso descentralizada (a través de las Agencias de Desarrollo Local) de entender, hacer y promover Desarrollo Local. Como afirma Juan Urrutia "Lo descentralizado implica grandes dosis de gestión", algo que ahora es cuanto menos inconcebible por cualquier administración.

Como conocedores que somos de los foros de Desarrollo Local, en muchos de ellos hemos percibido una cierta desazón motivada en gran parte por la inestabilidad del puesto de trabajo, el ciclo político que sufre el ADL, la dependencia del liderazgo del político de turno, la falta de visión de muchas administraciones locales que prefieren emplear a su ADL en temas de "gestión de subvenciones", "administrador", "apoyo a la secretaría", "chico para todo", etc.; en detrimento del papel de "dinamizador", "empoderador", "activador" que le sería mucho más adecuado. Para nosotros, esto solo hace que confirmar 
lo potente de un Desarrollo Local más distribuido que implica reflexionar sobre el potencial desarrollo de los ADLs en un ámbito privado y no sólo público, apoyado no tanto en una concejalía, sino en los agentes sociales (personas) del entorno.

Con esto no queremos decir que el Desarrollo Local distribuido no esté exento de dificultades, pero es más autónomo, empoderante y, lo más importante, más resiliente. En un modelo de Desarrollo Local distribuido, si el Ayuntamiento de un municipio no puede asumir la existencia de una Agencia de Desarrollo Local, la eliminación de este nodo no implicaría que se deje de hacer Desarrollo Local en ese municipio.

Por tanto, hablamos no tanto de un Desarrollo Local donde los ciudadanos, organizados o no, participan en el gobierno de un territorio, sino, de un Desarrollo Local donde los ciudadanos proponen y conversan con la administración en una interacción constante y sostenida en el tiempo. Ya no hablamos de redes participativas sino de redes de interacción entre nodos.

\subsection{La administración pública como un nodo más}

En este modelo distribuido la Administración es, como cada nodo, un agente generador de Desarrollo Local. Y lo es porque desde sus planteamientos y acciones (políticas de producción, de empleo, de crecimiento) actúa y piensa desde el aprovechamiento de recursos a su alcance, para asegurar la viabilidad y la sostenibilidad de esos recursos, de sí mismas y de su entorno/contexto. Y así generar abundancia. Al tiempo que ofrecen una interacción cercana, y por eso va desarrollando valores añadidos, que van encajando más, con las necesidades o los deseos de sus clientes. Y esto es independiente de su ubicación geográfica en un término municipal o en el mundo.

En términos de empresa privada, las pymes y micropymes, son agentes de este desarrollo, no por su localización, tamaño o alcance. Sino por su vinculación al territorio (contexto) y por su implicación con las personas (interlocutores directos). Por ejemplo, en muchos casos una multinacional localizada en un pueblo puede crear puestos de trabajo en ese territorio pero no genera riqueza/abundancia en él. Porque no favorece la diversidad, no valora las potencialidades ni las fomenta y no es flexible al cambio. En definitiva, no es resiliente. Ante los cambios que pueda experimentar el entorno dónde se ubica, esa multinacional, abandona el territorio o fuerza un cambio en el entorno que le sea propicio.

En esta nueva "topología de red" los lugares deben desarrollar "el capital social capaz de atraer y desarrollar talento e integrarlo en un espacio urbano, económico y de socialización cohesionado." (Ugarte D :2010). Y entendemos que esto sólo puede suceder si la relación entre los diferentes actores del desarrollo local y la administración es de colaboración, no sólo de negociación $\mathrm{o}$ asistencia. 
Por tanto hay una serie de cuestiones que la Administración debería plantearse en esta nueva topología de red. ¿Quién (o quiénes) es mi interlocutor? ¿cómo quiero hablar y cómo converso? ¿con qué tono, con qué medios? ¿cuáles son mis redes/relaciones de confianza? Y, previamente, debe pensarse a sí misma. Para poder mostrarse-narrarse y construirse realmente como un nodo conectado a otros por conexiones útiles. Para lo cual no debe olvidar el alcance que le permiten las tecnologías.

Estaríamos ante una modificación de las dinámicas de la administración actual, que pasaría de ser un emisor de información o servicios, a un ser un propiciador de recursos no siempre necesarios. Su rol en el desarrollo local puede ser de colaborador, de promotor o de beneficiario. Incluso quedando al margen de algunas acciones de desarrollo local iniciadas, ejecutadas y disfrutadas por otros nodos, ya sean estos individuos o colectivos. Y el criterio para ejercer uno u otro rol es resultado de la conversación y del vínculo con el entorno.

En este punto nos parece interesante el concepto, y el hecho, del "hermanamiento de ciudades". Las formas, objetivos y resultados, no vamos a discutirlas aquí y ahora. Pero es interesante detectar como la Administración local, entendió o quizás solo intuyó, la importancia y el potencial de una red distribuida de iguales. Y entendió que el tamaño y la ubicación no tenían porqué condicionar el alcance.

Así pues los mapas de vínculos para la planificación de las relaciones con el exterior de las organizaciones, que se nos ofrecen desde la disciplina de Relaciones Públicas; se rediseñan continuamente porque las relaciones varían según el rol que cada nodo puede ejercer para una acción concreta.

En este nuevo modo de relacionarse de la administración no podemos dejar de destacar la importancia de hablar un mismo lenguaje y compartir objetivos, para que la implicación personal no se desvanezca y poder trabajar en equipo. Pasar de "trabajar para" a "trabajar con", para lo que hace falta saber relatarse y conversar con los otros agentes.

\subsection{La visión hipercontexto del Desarrollo Local}

Por nuestra experiencia vital propia, nuestra manera de vivir y trabajar, podemos considerar que las personas ya no vivimos en un único contexto local geográfico, sino conectadas a muchos otros contextos locales, geográficos o no, gracias a lo digital. Tratar de convertir esta realidad en una oportunidad para el desarrollo local es uno de los modos de hacer que caracterizan a los profesionales de la red eGruyere.

Por ejemplo, lo local en nuestro trabajo es lo cercano a cada persona, no sólo ni principalmente lo cercano físicamente, sino aquello con lo que mantiene vínculos, con lo que se identifica, lo que forma parte de las interacciones de su vida cotidiana, lo que le permite desarrollar sus necesidades o inquietudes, 
preferiblemente desde sus afinidades y recursos. De ahí que uno de sus ejes principales en nuestros trabajos sea detectar esas afinidades, reales, concretas, que ya existen en las personas, así como sus recursos y potencialidades, diseñando los entornos para que las puedan, podamos, desarrollar.

La forma de diseñarlos y construirlos es haciendo uso de herramientas y metodologías colaborativas, porque es la forma en la que, como se ha explicado en los anteriores epígrafes, consideramos que puede tener lugar un desarrollo local distribuido y resiliente. Estos entornos se diseñan y construyen para que cada nodo, cada persona, pueda elegir y experimentar múltiples roles, es decir, ser múltiples agentes a la vez, dentro de dicho contexto (unas veces maestro, otras aprendiz, unas veces habitante, otras constructor, unas veces espectador, otras guionista...). Esta es la manera en que nos parece que se puede conseguir que las personas tengamos conciencia y actitud de ser agentes de desarrollo local de nuestros contextos o entornos cercanos.

El cambio que esta visión produce es que el nodo de acción del desarrollo local son las personas que conectan territorios, físicos o no, y que nosotros hemos dado en llamar 'hipercontextos'. El territorio y sus recursos se convierten entonces en un nodo más.

Pero ¿Por qué y Cómo pasar de la visión territorial geopolítica a la de hipercontexto? Para muchos autores la única posibilidad de desarrollo (incluso supervivencia) de un territorio es que supere la visión de la sociedad industrial para pasar a la de la sociedad del conocimiento. Esta sociedad está formada, para nosotros, por personas que habitan y se mueven en y entre hipercontextos.

En el texto "Nuevos Territorios", Juan Urrutia comienza hablando sobre la necesidad de de-construir el concepto de territorio describiendo un nuevo tipo de comunidades:

“...hay comunidades, (llamémosle convivenciales) que pueden ser desterritorializadas...

el mundo camina - a causa de las TIC, la sociedad de la información y la globalización - hacia un confederacionismo generalizado, global...en el que las comunidades identitarias desterritorializadas jugarán un papel de barreras o cortafuegos contra la propagación de peligrosos incendios..." (referencia bibliográfica)

Por eso, más que partir de lo territorial, a nosotros nos sirve partir de lo cercano frente a lo ajeno. Porque lo territorial geopolítico tiene el peligro de los localismos y del endemismo, cuando, para salir de situaciones estancadas, lo necesario es interactuar con lo extraño y encontrar las conexiones que construyan nuevos objetivos. Debemos hablar de nuestros entornos próximos $\mathrm{y}$, aprovechando las oportunidades que las tecnologías nos brindan, "explorar y emprender nuevos modelos de gobernanza, factibles en el actual contexto tecnosocial" (Torres y Santa, 2011:50) 
Toda esta de-construcción del concepto de territorio no es una amenaza para el desarrollo local de los propios territorios físicos, sino una ventaja, porque éste es "accionable" con pocos medios y desde la pequeña escala, donde importan más las personas.

\subsection{Un entorno concreto, no universal}

Observamos que la mayoría de visiones o propuestas de DL se relacionan con cierta mirada sobre el mundo, y en especial sobre los recursos naturales, patrimoniales, etc., que dificulta implementar la forma de generar desarrollo local que presentamos. Una mirada universalista que nos hace ver que hay ciertas cuestiones que atañen a todo el mundo y que se puede pensar en ellas con la idea de un bien común universal en el que todos estaríamos de acuerdo. Para terminar la comunicación, explicaremos por qué esta mirada dificulta nuestra forma de generar desarrollo local y expondremos nuestra mirada sobre el entorno, el medio ambiente y el patrimonio.

Resumiendo nuestra propuesta, hablamos de que varios agentes o actores diversos se alíen entre ellos con nuevos modelos de gobernanza, donde el peso y la importancia les dan la capacidad de tener ideas, encontrarles financiación y llevarlas a cabo, para generar proyectos concretos en un entorno local que hemos llamado hipercontexto. Ante esta propuesta pueden aparecer, al menos, dos reticencias:

- Que algunos de los agentes entiendan que tienen más o menos poder que otros sobre un recurso concreto del entorno, basándose en atribuciones burocráticas, fundamentadas en fronteras, naciones, etc., en lugar de en función de la cercanía que hemos descrito en el epígrafe anterior.

- Que algunos de los agentes intenten imponer sus ideas sobre las del resto basándose en ideas universalistas sobre lo que es bueno, en lugar de intentar negociarlas según sus necesidades concretas.

Ya hemos introducido la idea de que, para generar desarrollo local, no podemos pensar en fronteras administrativas, sino en hipercontexto. Esto es especialmente evidente cuando trabajamos con recursos patrimoniales o, lo que se ha llamado, bienes comunes (naturales, artificiales o de conocimiento, no entraremos en esa distinción). Porque estos recursos normalmente serán transfronterizos, sus afecciones traspasarán las líneas imaginarias que se han trazado en los territorios. Entonces ¿cómo saber nuestros derechos y responsabiliades? Para responder a esta pregunta seguiremos con la idea de que el contexto hiperlocal es el que nos afecta en nuestra vida. O como propone Tim Ingold, antropólogo inglés, sabremos nuestros derechos y responsabilidades "a través de nuestras propias prácticas de habitar" (Ingold 2011:114).

Las prácticas de habitar no son las prácticas de consumir (a las que no empuja la idea de consumo responsable), sino las de producir nuestro entorno en nuestro "campo de prácticas" o "espacio de acción", que es el espacio donde 
podemos actuar con nuestras habilidades, materiales y herramientas, que no necesariamente es un espacio pequeño en sentido territorial.

Pero para ser coherentes con esta idea, y evitar la segunda posible reticencia a nuestra forma de generar desarrollo local, también renunciamos a pensar que hay acciones sobre los recursos que son inherentemente buenas o que hay recursos que afectan a todos. Estas dos ideas son bastante frecuentes cuando se trabaja con recursos del medio ambiente y puede ser polémico rebatirlas. Nuestra propuesta incluye la negación, en la práctica, de un mundo global y del famoso eslogan "piensa global, actúa local". Preferimos pensar que es un mundo esférico, que nos envuelve. Porque "la diferencia entre la perspectiva 'local' y 'global', no es una jerarquía de grado, en escala o comprensión, sino de clase. En otras palabras, lo local no es una manera más limitada o estrecha de aprehensión que lo global, es una que se soporta en un modo completamente diferente de aprehensión - un modo basado en un compromiso práctico y perceptivo con los componentes de un mundo que es habitado, más que una observación separada y desinteresada de un mundo que es simplemente ocupado" (Ingold 2000: 215-216).

No decimos que el mundo no sea un globo, ya sabemos que es así visto desde el espacio exterior. Sino que desde el espacio de acción de los agentes que intervienen en el desarrollo local el mundo es más bien una esfera alrededor de estos agentes. En el mundo global los problemas son de una escala inaccesible para estos agentes, en el mundo esférico los problemas son de la escala a la que estos agentes pueden actuar.

Por tanto, ya no deberíamos pensar si lo que hacemos es bueno para los ríos, los bosques, los animales, la memoria histórica,... en abstracto. Sino que nos preguntaremos si es bueno para el mantenimiento de nuestra comunidad que incluye (dentro de su esfera o su campo de acción) unos recursos en concreto. Recursos con nombres y apellidos, igual que las personas con las que trabajamos. Porque son espacios o recursos con los que trabajamos para el mantenimiento físico de nuestra comunidad $\mathrm{y}$, por eso, los cuidamos. Manteniéndolos en el estado en que continúan dándonos lo que necesitamos o modificándolos para que lo hagan. No sólo desde un punto de vista económico sino también simbólico, porque eso es también parte de nuestra comunidad. Y no sólo recursos tangibles, sino también intangibles.

Es decir, limpiar un río puede ser bueno para una organización o comunidad, pero quizá no lo sea para otro agente. Entonces, de todos los agentes que comparten, respecto a ese río, un espacio de acción, lo que hemos llamado un hipercontexto, me convendrá asociarme con los que también consideren que limpiar ese río es bueno. Y las razones por las que ambos agentes lo consideran bueno no pueden imponerse per se a las del resto. Serán necesarias las negociaciones propias del modelo de acción distribuido con el que hemos empezado esta comunicación. 
Si no fuese así, si apeláramos a la idea de que limpiar el río es universalmente bueno porque es un recurso global que hay que mantener limpio porque a todos nos afecta, volveríamos a salir disparados al espacio exterior y perderíamos toda nuestra capacidad de acción sobre ese recurso. Y, por tanto, perderíamos toda la capacidad de generar desarrollo local de la forma que hemos presentado.

También perderíamos esta capacidad si, por otro lado, permitiéramos a algún agente apelar a su poder abstracto sobre ese río. Por ejemplo, con un antiguo derecho de uso de aguas del que ya no se hace uso pero que le hace mantener la jurisdiscción. Si permitimos que un agente que no usa ese recurso (o que no hace explícita la forma en que lo usa) ejerza ese tipo de derechos abstractos, perdemos la capacidad de negociación y generación de alianzas que es la base de esta propuesta. Aunque ambas cosas ocurren y ocurrirán a menudo, y seguimos trabajando en ello.

Este conjunto de visiones, que estamos practicando en eGruyere contribuye a la resiliencia de las personas y sus contextos locales. Desde nuestras empresas, cooperativas, asociaciones, etc., estamos convirtiendo problemas en oportunidades y desarrollando nuevas propuestas en respuesta a dichos hipercontextos, para lo que es imprescindible una actitud creativa.

\section{Casos de estudio}

\subsection{Proyecto 1: Propuesta de Rehabilitación Integrada del conjunto Minas de San Antón.}

\subsubsection{Desarrollo local distribuido:}

Esta es una propuesta de desarrollo local a través del proceso de rehabilitación integrada y posterior uso autogestionado de un bien inmueble público, considerado patrimonio arqueológico, situado en el barrio de San Antón, Orihuela. La propuesta es planteada desde la organización no gubernamental Proyecto áSILO, cuyos trabajos se centran en el desarrollo de oficios necesarios para reconstruir los entornos que habitamos. Al conocer dicho trabajo algunas personas integrantes de la concejalía de medioambiente y patrimonio de Orihuela, nos formulan la pregunta: ¿Cómo rehabilitaríais este bien inmueble, propiedad pública, y su entorno natural inmediato, desde los modos de hacer de los SILERUS?

Entendimos que les interesaban de estos proyectos, los procesos por los que se llevaron a cabo y los resultados finales tangibles e intangibles que dieron. Por tanto, nuestra propuesta sería un proceso.

Era necesario definir los objetivos principales de nuestra propuesta: No es un objeto final, es un proceso de aprendizaje, experimentación y reactivación de una comunidad local, por cuanto afín al contexto de trabajo, que dará como resultado físico la rehabilitación y transformación de una ruina en un bien co- 
mún. Para lo cual las preguntas importantes son QUIENES Y CÓMO primero, y a través de QUÉ después.

Para nuestro planteamiento es fundamental abrir los procesos a la iniciativa ciudadana existente y provocar la interacción con nuevos grupos interesados, llegando a formar equipos entre agentes locales y agentes externos o glocales que deseen trabajar conjuntamente para llevar a cabo el proyecto, integrando los recursos propios y potenciales del contexto.

La Administración como un nodo más

No fue un ente abstracto, una concejalía, la que se interesó por nuestro trabajo, sino tres personas, con nombres y apellidos, que trabajan en dicha concejalía, con inquietudes acerca de cómo reformular los procesos para el desarrollo de una localidad. Vieron en nuestro trabajo un compromiso por investigar con nuevas metodologías, herramientas, pero siempre potenciando los recursos propios.

Al plantearles la propuesta, quisimos hacerles entender que la única forma posible de llevar a cabo este proyecto es haciendo un equipo que sea capaz de trabajar colaborativamente, pudiéndose construir así esa confianza necesaria para que procesos como el planteado sean posibles, permitiendo la experimentación intencionada y programada, motor de descubrimiento e innovación.

Por eso, la tarea primera que tuvieron que realizar desde la administración, fue encontrar el tipo de contrato que, acorde a la ley de contratos del Sector Público, un servicio así requiere.

Tras conversaciones con los diferentes agentes administrativos encargados de ello, el único consenso era que no se trataba de un contrato de obras sencillamente porque nuestra labor no es únicamente la realización de una obra de construcción.

Investigamos con la posibilidad de realizar un "Contrato de colaboración entre el sector público y el privado", puesto que su descripción encaja en lo que estamos proponiendo, no únicamente porque aparezca la palabra colaboración, sino porque es una actuación global e integrada, con desarrollo de talleres y oficios, investigación en servicios y productos con tecnologías situadas para rehabilitación, gestión colectiva de bienes públicos....

Pero el Secretario consideró que este tipo de contratos se destinan a propuestas de I+D, de altisimos presupuestos.

Se llegó al acuerdo de hacer un "Contrato de servicios", para redactar y construir las herramientas que posteriormente permitirían llevar a cabo este proyecto.

\subsubsection{La visión hipercontexto}

Nuestro concepto de "desarrollo" está enfocado a descubrir la abundancia de oportunidades y servicios que un bien común puede generar desde su propio 
proceso de rehabilitación. La comunidad local que estamos formando no es endógena de un territorio, sino que podrá estar formada por todas aquellas personas, que viviendo o no físicamente cerca del objeto de trabajo, establezcan algún tipo de vínculo con él, ya sea en todo su proceso, o en una parte.

\subsubsection{Un entorno concreto}

Consideramos que son las comunidades locales las que han de gestionar aquello que identifican como patrimonial. Nosotros remarcamos la importancia de que sean ellas las que definan si algo, ya sea material o inmaterial, es un bien común para ellos, y en qué medida podrán beneficiarse de gestionarlo entendiendo ese beneficio como una oportunidad real de desarrollo local. Por tanto nuestra labor primera será la de fomentar las preguntas y dinámicas que lleven a dicha comunidad a posicionarse.

\subsection{Proyecto 2: Ruta Emprende Artesana}

\subsubsection{Desarrollo local distribuido}

Esta es una acción diseñada y desarrollada por la iniciativa de colaboración de empresas privadas, en concreto tres profesionales de diferentes disciplinas (Arquitectura y Comunicación). En un primer momento el proyecto se ha ejecutado en colaboración con la administración local. Esta dirigido especialmente a mujeres, pero también hombres, con habilidades y aficiones, más o menos artesanales, y acotadas al ámbito doméstico, por lo que no son visibles. Pero que pueden ser convertidas en actividad económica y/o propiciar la autoafirmación de las artesanas.

Este proyecto se plantea para dar visibilidad a ciertas habilidades o aficiones, especialmente femeninas, que no son valorados o son invisibles, así como sus "hacedoras". Y que al no ser visibles, no tienen la posibilidad de profesionalizarse, de aportar ingresos extras y/o servir de autoafirmación para quienes las realizan. Entendemos que estas personas tienen un gran valor para el territorio que habitan, pero que actúan como agentes invisibles y latentes del desarrollo de su entorno. Además el taller se ocupa de ciertos aspectos fundamentales, previos a la puesta en marcha de un negocio, y que exigen de la persona emprendedora, procesos y planteamientos concretos.

Financiada por la entidades colaboradoras, empresas y administración, y las aportaciones de los usuarios finales.

\subsubsection{La Administración como un nodo más}

Para la puesta en marcha del primer taller la Administración aceptó como propios los objetivos del proyecto, ya que encajaban en sus propios objetivos y políticas y las materializaba en una acción concreta. 
Aportó recursos materiales básicos, espacio e infraestructura. Se responsabilizó de publicitar y hacer llegar la información a los públicos específicos (mujeres con gap tecnológico pero con habilidades artesanales), con los que tiene diálogo directo.

\subsubsection{Contexto hiperlocal}

El proyecto ejecutado es un taller de cuatro sesiones. El proyecto diseñado es una ruta con diferentes "paradas", los talleres. Dicha ruta y el conocimiento generado en cada parada está disponible en un blog y en un mapa que refleja el trabajo cotidiano y la diversidad artesana, y que acabará funcionando como una comunidad. Una comunidad útil tanto para las artesanas como para su entorno. Que genera una red virtual de municipios conectados por una ciudadanía emprendedora, por sus actividades, por sus saberes y sus aspectos comunes.

Permite a sus miembros estar conectados y visibles en lo digital. Lo que supone cierto grado de empoderamiento.

Permite a los participantes estar acompañados en determinados procesos imprescindibles para la puesta en marcha de un negocio. Desde definir aquello que les apasiona; hasta identificar las posibles barreras y la forma de superarlas. Pasando por reconocer el entorno, tanto local como digital en el que se encuentran; o el mercado al que se dirigirán, entre otros.

\subsubsection{Un entorno concreto (qué recursos y cómo es importante para cada quien)}

Es un proyecto formativo y de asesoramiento, que permite iniciar un proceso de crecimiento profesional en compañía, con personas en su misma situación y con guías que ya están activando sus pasiones. Por tanto el recurso que se pone en valor son las propias personas artesanas, con nombres, apellidos y pasiones concretas.

Los participantes van definiendo los recursos de su entorno que son valiosos para ellas y los marcan en un mapa, de forma que su actividad y su entorno se ven mutuamente favorecidos.

Por otro lado, toda la programación de la plataforma y las herramientas que las artesanas aprenden a usar utilizan software libre que nos (y las) independiza de licencias prohibitivas y, sobre todo, hace que pasen a formar parte de las comunidades de usuarios de este software que, al contrario que el software privativo, nos permite involucrarnos en su desarrollo. Como los recursos locales, este software pasa a formar parte del entorno concreto de estas artesanas porque les permite realizar su actividad más libremente. 


\subsection{Proyecto 3: Mas del Planet: Centre Rural d'Exerimentació. http://masdelplanet.es/}

El Mas del Planet es un Centro cultural sobre su entorno, el mundo rural. Un lugar donde compartir conocimientos, experiencias, ideas y nuevos proyectos mediante cursos, exposiciones, charlas, trabajos colaborativos y otras propuestas de utilidad social. Su objetivo principal es experimentar con nuevas formas de vivir en el mundo rural. Está situado en La Torre de les Maçanes y las principales áreas de experimentación son: nuevas tecnologías, agricultura, bioconstrucción y desarrollo económico rural. La primera área que se está trabajando es la bioconstrucción con la rehabilitación del Mas en forma de talleres de rehabilitación patrimonial y bioconstrucción.

\subsubsection{Desarrollo local distribuido}

El proyecto está promovido por los propietarios del Mas, asesorados por una de las micropymes de eGruyere, aRRsa! Plataforma Creativa. Pero en él participan varias asociaciones y empresas locales, como la asociación ecologista El Rentonar, la empresa SócdePoble SL, y colaboran varias empresas, personas y asociaciones de otros municipios, todas vinculadas al mundo rural. También se está trabajando con Departamentos de investigación de universidades. De hecho, este proyecto es posible porque los propietarios del Mas son miembros activos de estas asociaciones y empresas y todos juntos entienden que un proyecto así es dinamizador de la economía y la sociedad de ese entorno rural. Pero no de una forma abstracta, sino que cada persona y organización involucrada ve sus beneficios en este proyecto. Los propietarios del Mas tendrán una nueva fuente de ingresos que, aunque pequeña, los diversifica y aporta resiliencia, además ven mejorado su patrimonio. Las asociaciones locales amplían su oferta de actividades y, en el futuro próximo tendrán un nuevo espacio donde desarrollarlas por un acuerdo con los propietarios. La empresa SócdePoble (que ofrece nuevas tecnologías desde y para el mundo rural) tendrá aquí su sede y una fuente de contactos y posibles nuevos clientes. El resto de organizaciones se benefician también por el efecto red. Y todos los participantes en talleres y actividades ganan en conocimientos diversificando también sus posibilidades de una vida digna en el mundo rural, ya sea aprendiendo nuevos oficios o reinventando los viejos.

\subsubsection{La Administración como un nodo más}

En este caso, aún no se ha colaborado con las administraciones, pero no se descarta. Presentamos este proyecto porque nos parece una muestra del potencial de desarrollo local desde la iniciativa privada y civil. La financiación principal proviene de los propietarios del Mas y el activo más importante, el conocimiento, proviene de las personas y organizaciones que se involucran. 


\subsubsection{Contexto hiperlocal}

Como hemos comentado, el contexto de este proyecto es, precisamente, su razón de ser. La reflexión y experimentación sobre nuevas formas de vida en el mundo rural. Las áreas que se experimentan son las que afectan en el día a día a las personas que viven en este entorno y las soluciones que se experimentan son comunes a los implicados y a los participantes. Por ejemplo, en la primera área que se está trabajando, la rehabilitación y la bioconstrucción, las soluciones propuestas están basadas en la arquitectura vernácula y se da especial importancia a las soluciones susceptibles de ser autoconstruidas. Además, se promueven las soluciones de infraestructura distribuida, por ejemplo los baños secos o la energía solar, y el uso de materiales locales como la caña, el barro, la piedra seca,... En el área de agricultura se trabaja con la agricultura local y ecológica adaptada al clima local y a los pequeños minifundios. En las nuevas tecnologías se trabaja con software libre y se estudian formas de interconexión adecuadas para el mundo rural.

\subsubsection{Un entorno concreto}

Los recursos con los que se trabaja son: el propio Mas, los materiales de la zona y el capital social y el capital de conocimiento de las personas y organizaciones que participan. Por ello, se hace especial hincapié en el formato de taller y en la divulgación de las experiencias se realizan.

Con este proyecto no se pretende aportar soluciones universales a la vida rural, sino aportar propuestas y experiencias concretas que, gracias a que la información es compartida, son susceptibles de reproducirse en contextos similares por personas que consideren que tienen necesidades y prioridades parecidas. Pero ninguna de las soluciones y propuestas pretende imponerse. Que la iniciativa sea privada es una prueba de ello. En lugar de imposición se trabaja por proposición.

\subsection{Proyecto 4: Tropos http://tropos.es/}

Tropos es un proyecto audiovisual cuyo objetivo es abrir la reflexión sobre la manera de contar las historias desde las herramientas contemporáneas y hacia la construcción de relatos futuros. Tropos nace a finales de 2011 con el teaser Equinoccio, un corto que pone la semilla que creará Tropos, una webserie de la que actualmente se ha rodado la Temporada 0 compuesta por tres capítulos de 8 minutos cada uno. Los Tropos son unos seres capaces de ver lo más íntimo del ser humano: sus corazones. Y pueden también modificarlos. 


\subsubsection{Desarrollo local distribuido}

La Temporada 0 de Tropos fue impulsada por 4 organizaciones juntando a un equipo de 24 profesionales. En la actualidad, el equipo de Tropos está formado en su núcleo por dos organizaciones: Reding Estudio Creativo y Ciudad de la Sombra.

\subsubsection{La Administración como un nodo más}

La Administración Pública ha tenido una participación puntual y concreta en el proyecto, concretamente haciendo posible la asistencia al rodaje de 4 alumnos del I.E.S. Azorín de Yecla mediante una beca concedida directamente por el Ayuntamiento de Yecla. Con esta beca los alumnos asistieron como espectadores al rodaje de Tropos durante el mes de junio de 2012.

A su vez, se colaboró con el Ayuntamiento de Alicante en la difusión del proyecto a nivel provincial, consiguiendo aparecer en televisiones provinciales.

En Yecla, se colaboró también con el Ayuntamiento de Yecla en la difusión del proyecto y presentación en rueda de prensa.

\subsubsection{Contexto hiperlocal}

Tropos es un ejemplo claro de la visión del contexto hiperlocal. Sólo en la primera temporada han participado un total de 24 colaboradores de diversas procedencias de España, todos ellos eso sí, muy centrados en el arco mediterráneo, desde Barcelona hasta Murcia, pasando por Valencia y Alicante. Profesionales que han dedicado su tiempo y conocimientos a poner en marcha este proyecto.

En el tema de la financiación, Tropos ha financiado la primera temporada vía crowdfunding, lo que significa que 90 personas dispersas geográficamente por todo el país han conocido el proyecto y han decidido apoyarlo económicamente.

Ahora en la segunda fase, estamos trabajando en la idea de trabajar con la pequeña comunidad que ha surgido en torno a Tropos, personas que puedan intervernir en él en encuentros físicos y desde herramientas online.

\subsubsection{Un entorno concreto}

Tropos no pretende relacionarse con todo el mundo ni llegar a todo el mundo, que sería en sí inabarcable, sino involucrar a personas con ganas de experimentar en el audiovisual y con cierto carácter amateur. Personas cuya motivación sea el aprendizaje y la visibilidad profesional, que quieran poner conceptos en práctica en los siguientes ábmitos: Guión, preproducción, financiación, iluminación, sonido, arte, interpretación, rodaje, montaje, banda sonora, etalonaje digital, difusión pública, estreno, distribución. E importante, personas para las que la remuneración no sea lo único o principal. 
Tropos apuesta por jugar con la fantasía, el documental y el experimental. Que la clave del producto final sea una narrativa más "documental" con dosis de "fantasía" donde se está "experimentando", pues se está aprendiendo mientras se realiza.

Y teniendo claras estas dos coordenadas, tenemos claros los agentes con los que queremos trabajar y las necesidades que deseamos cubrir.

\section{Conclusiones}

Concluiremos diciendo que nosotros estamos en el núcleo de nuestro desarrollo local. Estos conceptos expuestos y sus casos prácticos son el ejemplo de ello. Nuestro modelo de desarrollo local es distribuido, centrado en las personas y en los recursos locales, un modelo abierto, influenciable y en constante evolución. Entendido así, casi podemos llamar a estos nuevos agentes de desarrollo local como agentes de "abundancia" local.

Nuestro concepto de gobernanza va más allá del que actualmente se da en la mayoría de las instituciones y que considera ese acto, gobernanza, como una competencia propia de determinadas instituciones que aplican determinados modelos. Nosotros prestamos especial atención a la segunda acepción del término que da la RAE "Gobernanza :2. f. ant. Acción y efecto de gobernar o gobernarse." ...

Este conjunto de visiones, que estamos practicando en eGruyere contribuye a la resiliencia de las personas y sus contextos locales. Desde nuestras empresas, cooperativas, asociaciones, etc., estamos convirtiendo problemas en oportunidades y desarrollando nuevas propuestas en respuesta a dichos hipercontextos, para lo que es imprescindible una actitud creativa.

eGruyere hace Desarrollo Local distribuido en la medida en que: genera y apoya a empresas, que desarrollan sus conocimientos e intereses para la generación de abundancia en su entorno. Define sus herramientas, entre ellas, la ética hacker del trabajo, que nos invita a relatarnos y a construir la confianza necesaria para la colaboración. Y genera sinergias de aprendizaje y conocimiento que nos hacen más resilientes. eGruyere es para nosotros una incubadora de proyectos de Desarrollo Local y una propuesta de futuro para el Desarrollo Local.

\section{BiBLIOGRAFÍA}

AMASTÉ, R. (2013). "\#EUSKADI_P2P, el lugar más copiado del mundo" Colaborabora,

BELTRÁN PATOR, S. (2013), "Desarrollo local, pymes y creatividad. Notas I." Comunicación, Cultura y otros intereses. Ensayo y error de una profesión. http://soniabeltran.wordpress.com/

GISBERT ALEMANY, E. (2013), "Cap a un model distribuït d'acció en el territori: un medi ambient concret i carregat de significat" Colabores. 
Herramientas, metodologías y conceptos para la reconstrucción cotidiana del entorno. http://colabores.net/

INGOLD, T. The Perception of the Environment. Routledge. London. 2000

INGOLD, T. Being Alive. Routledge. Oxon. 2011.

JAVALOYES, M.(2013). "Hablemos de desarrollo local" Entornos desbordantes. http://desbordanteysinrigor.blogspot.com.es/

PÉREZ UGENA, A. (Coordinador). Relaciones Públicas: Aplicación práctica a las organizaciones privadas y públicas. Capítulo XIX. MATERIALES PARA LA INNOVACIÓN EN LA ESTRUCTURA DE LA COMUNICACIÓN. Colección Bolonia. Ciencias de la Comunicación. Editorial Universitas, SA. Madrid. 2011.

REY, A. "Un nuevo modelo socioeconómico: comentando a Ricardo Antón (post-354)" 05/01/2013

TORRES VALDÉS, R. M. (2008). Aplicación de instrumentos de Relaciones públicas en el ámbito del desarrollo local. Descripción y posibilidades. Tesis doctoral UAB. Disponible en: http://www.tdx.cat/bitstream/ handle/10803/4146/rmtv1de1.pdf?sequence $=1$

TORRES VALDÉS, R. M. y SANTA SORIANO, A (2010). Principios y técnicas de Relaciones Públicas en la estrategia de Desarrollo Local. Manual de Estudio. Disponible en http://www.escuelagobierno.org/escuela/ index.php?sub_cat=25982

UGARTE, D. (2010). Un modelo indiano de desarrollo local. Disponible en http://bitacora.lasindias.com/un-modelo-indiano-de-desarrollo-local/

URRUTIA , J. (2012). Nuevos Territorios. encuentro Basques 2.0 Fundazioa. Egino (Araba),

VON BUSCH, O. (2008). Fashion-able. Hacktivism and Engaged Fashion Design. School of Design and Crafts (HDK) Faculty of Fine, Applied and Performing Arts.

YAGO MARTÍNEZ, L. (2011-2013). Innovación relacional. Estrategias digitales en el ámbito local. (Categoría "Desarrollo Local") http:// innovacionrelacional.somosreding.com/ 